\title{
La diabetes gestacional en embarazadas que reciben atención ambulatoria
}

Aída Dolores Aceituno López, Hessylda del Pillar Elvir Aguilar, Sairy Celeste Ordóñez Vásquez ${ }^{1}$

Perla Simons Morales ${ }^{2}$

\section{RESUMEN}

El objetivo primordial de esta investigación es determinar el nivel de conocimiento sobre diabetes gestacional, conocer cuáles son los factores de riesgos más conocidos por las embarazadas e identificar los alimentos de mayor consumo en las embarazadas que reciben atención ambulatoria en el área de ginecología del Centro de Salud Alonso Suazo.

La investigación se basó en una metodología de tipo cuantitativo, corte transversal; ya que la recolección de datos se dio en un tiempo único, con un alcance descriptivo. El universo fue de 22 embarazadas, de las cuales 15 fueron tomadas como muestra y 6 piloto. Los criterios de inclusión y unidad de análisis fueron todas las embarazadas ambulatorias que reciben atención ambulatoria en el Centro de Salud Alonso Suazo. De igual manera, los criterios de exclusión y eliminación fueron las mujeres que no están embarazadas y que no reciben atención en el área de ginecología del Centro de Salud Alonso Suazo.

Como resultado de la investigación realizada se observó que el 80 \% de las embarazadas que asisten al Centro de Salud Alonso Suazo no tienen conocimiento sobre qué es la diabetes gestacional, lo cual permite tomar conciencia de la gravedad del problema, promover nuevas estrategias de prevención y control sobre esta enfermedad, tomando en cuenta que una dieta sana, equilibrada y baja en carbohidratos, puede prevenirles de padecer diabetes gestacional.

Palabras claves: diabetes gestacional, conocimiento, prevención, estrategias, atención ambulatoria.

${ }^{1}$ Estudiantes, Escuela de Enfermería, Facultad de Ciencias Médicas, UNAH: ayessy@yahoo.es

${ }^{2}$ Asesora, Escuela de Enfermería, Facultad de Ciencias Médicas, UNAH: simonsperla@yahoo.es 


\section{ABSTRACT}

The main goal of this research, is to determine the level of knowledge about the stages of diabetes in pregnant women, knowing the mayor risks factors, and also identifying the most consumed foods by pregnant women who receive ambulatory attention in the gynecology station at the Alonso Suazo health center.

This research is based in a quantitative methodology, cross-section design , because the data was collected in a certain time, obtaining a descriptive scope. The population in study were 22 pregnant women, 15 of them represent the main sample and 6 of them were considered the pilot sample. The inclusion criteria and analysis unit were all the pregnant woman who receive ambulatory attention at the Alonso Suazo health center. In the same way the exclusion and elimination criteria used in this study was the number of women who were not pregnant or those women who have not received attention in that gynecology station.

This research determined that $80 \%$ of the pregnant women who attended the health center have no knowledge about gestational diabetes and that allowed us to have awareness of the seriousness of this problem. The research intends to promote new strategies for prevention and control of gestational diabetes, considering a balanced and low carbs nutrition as means of prevention.

Key words: gestational diabetes, knowledge, prevention, strategies, ambulatory attention. 


\section{INTRODUCCIÓN}

Según la OMS (2015), la diabetes mellitus es el tercer problema de salud pública más importante en el mundo y la diabetes gestacional es parte de uno de los 4 grupos de esta patología.

La diabetes gestacional se define como una intolerancia a los carbohidratos de severidad variable, que comienza o es detectada por primera vez durante el embarazo (Campo y Estrada, 2008). Tal enfermedad se caracteriza por la hiperglucemia (aumento de azúcar en sangre), razón por la cual estas mujeres corren mayor riesgo de sufrir complicaciones durante el embarazo y el parto, además de padecer de diabetes tipo 2 en el futuro (OMS, 2015).

En la actualidad se clasifica a las gestantes en dos grupos según su riesgo de desarrollar diabetes a lo largo del embarazo:

1. Gestantes de alto riesgo: son aquellas que presentan uno o más de los siguientes factores: edad mayor de 25 años, antecedentes personales de diabetes gestacional, antecedentes diabetes mellitus en familiares de primer grado, excesivo aumento de peso en el $2{ }^{\text {do }}$ y el $3 .{ }^{\text {er }}$ trimestre (IADPSG, 2010).

2. Gestantes de riesgo moderado bajo: son todas las que no tienen ninguno de los factores de riesgo anteriores.

A diferencia de los otros tipos de diabetes, la gestacional no es causada por la carencia de insulina, sino por los efectos bloqueadores de las hormonas en la insulina producida, una condición denominada resistente a la insulina, que se presenta a partir de las 20 semanas de gestación. Según proyecciones de la OMS, la diabetes será la séptima causa de mortalidad en el 2030 (OMS, 2015).

En este sentido, determinar los conocimientos sobre la diabetes gestacional en las embarazadas que reciben atención ambulatoria permitirá conocer la situación del problema y tomar medidas que contribuyan a un mejor control preventivo. 


\section{METODOLOGÍA}

La metodología utilizada en esta investigación fue de tipo cuantitativo, de corte transversal, ya que la recolección de datos se hizo en un periodo de tiempo único (abril-mayo), con un alcance descriptivo, utilizando la encuesta como método para la recolección de datos.

La muestra fue intencionada tomando a todas las embarazadas presentes el día que se hizo el levantamiento de la información, las cuales se encontraban a la espera de recibir sus controles médicos correspondientes en la sala de ginecología del Centro de Salud Alonso Suazo.

En el análisis estadístico los programas utilizados para obtener los resultados fueron: SPSS y Excel. De igual manera, se sacaron tablas de frecuencia, gráficos circulares, gráficos de barra e histogramas.

Con respecto a las normas de ética se solicitó permiso por escrito a las autoridades del Centro de Salud Alonso Suazo (específicamente al director Dr. Luis Largaespada). Asimismo, se observó la primacía del sujeto, pues se mantuvo el respeto y la dignidad de las embarazadas.

Participación voluntaria, libre e informada de los sujetos: a todas ellas se les explicó y se les presentó la encuesta, sin obligarlas a participar.

Garantía del derecho a la intimidad de los sujetos: se mantuvo en estricta confidencialidad con la información recolectada.

\section{RESULTADOS}

Según el gráfico 1, la mayor parte de las mujeres embarazadas (12) encuestadas no tienen conocimiento sobre la diabetes gestacional, concluyendo que un porcentaje mínimo (3) es el que conoce un poco sobre el tema en referencia. 
Gráfico 1. Conocimientos sobre diabetes gestacional

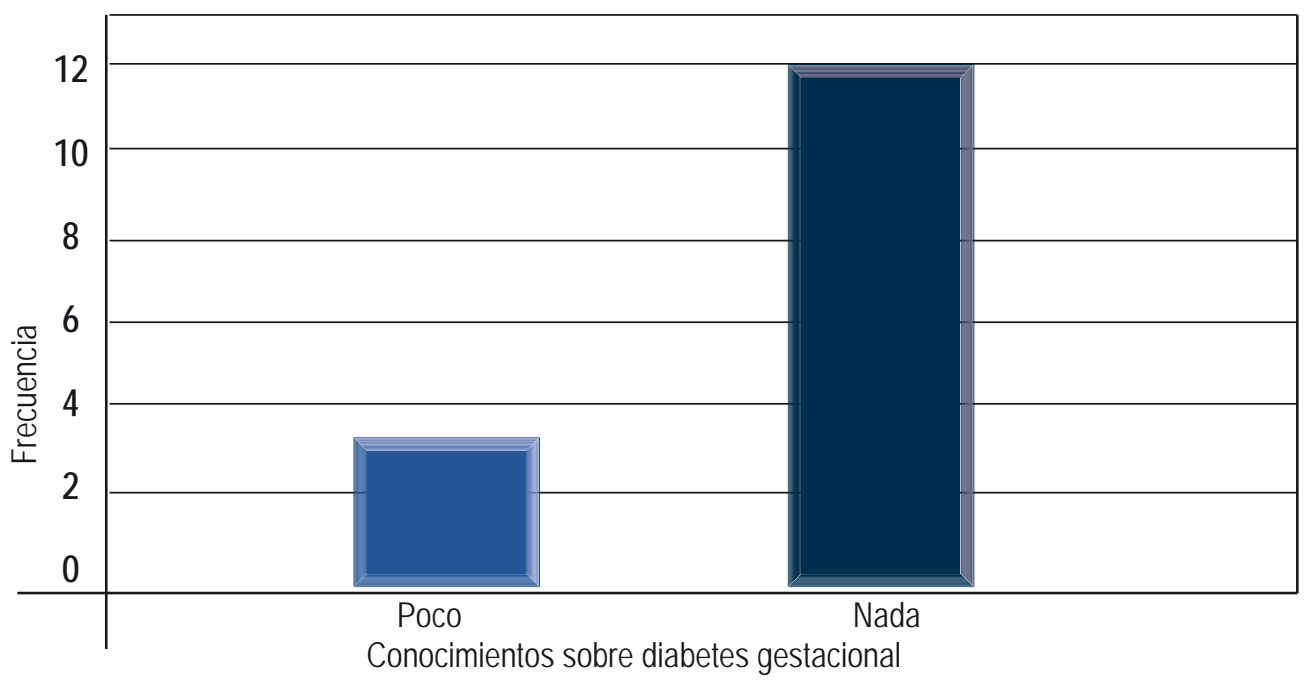

Fuente: elaboración propia.

Gráfico 2. Factores de riesgo del desarrollo de diabetes mellitus, según las pacientes

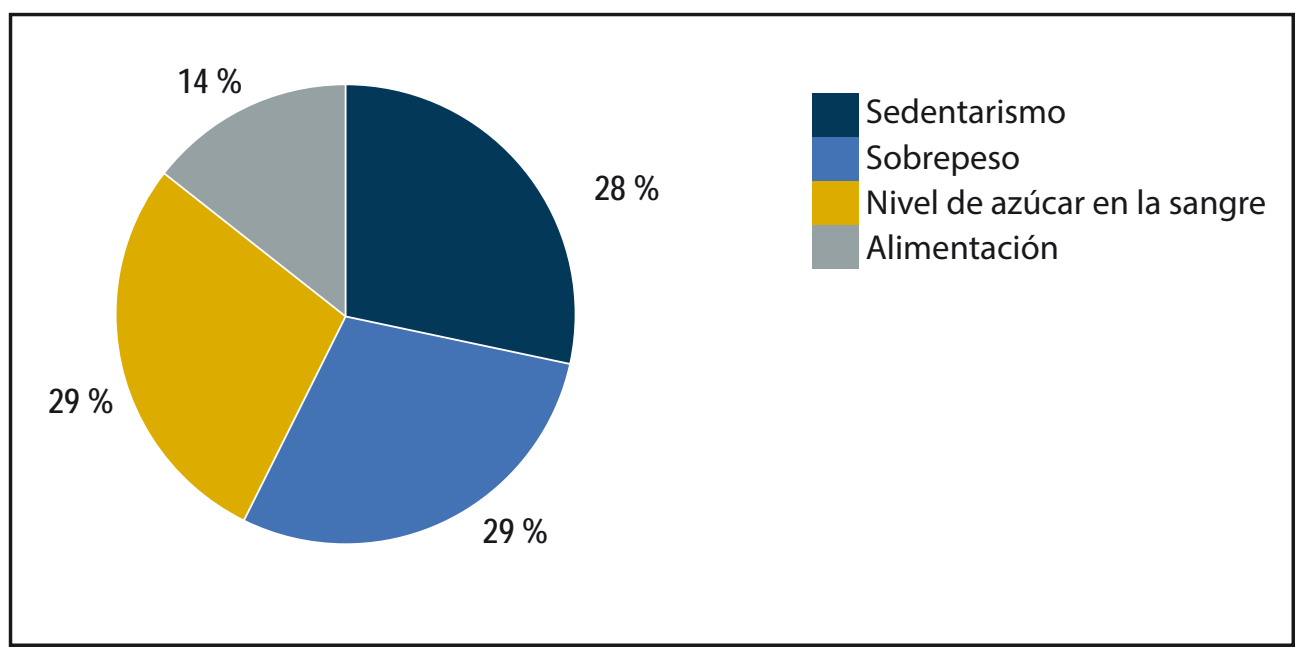

Fuente: elaboración propia.

El gráfico 2 indica que, según las opiniones brindadas por las embarazadas, el nivel de azúcar en sangre y el sobrepeso son los principales factores de riesgo para desarrollar diabetes mellitus, obteniendo así un mayor porcentaje. Pero, además de estos dos también hicieron mención de la alimentación y el sedentarismo como parte del conjunto de factores de riesgo. 
Gráfico 3. Caracterización de las embarazadas

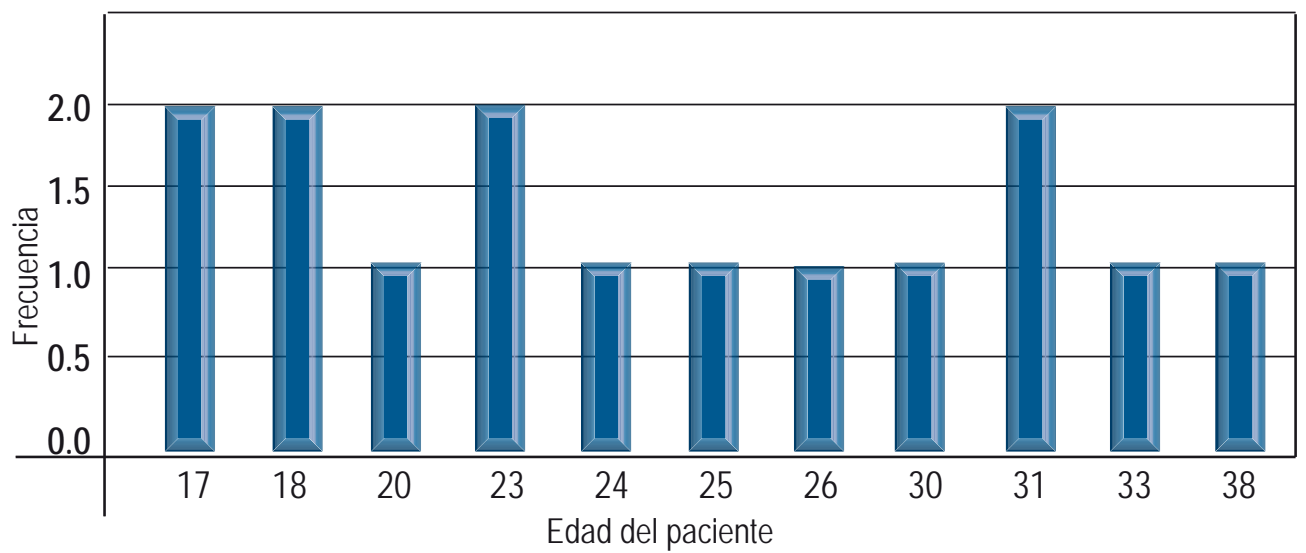

Fuente: elaboración propia.

Según el gráfico 3, la edad de las embarazadas que reciben atención ambulatoria se ubican en un rango de 17 a 38 años de edad, predominando las siguientes edades: 17-18 y 23-31 años.

Gráfico 4. Peso de las embarazadas en kilogramo

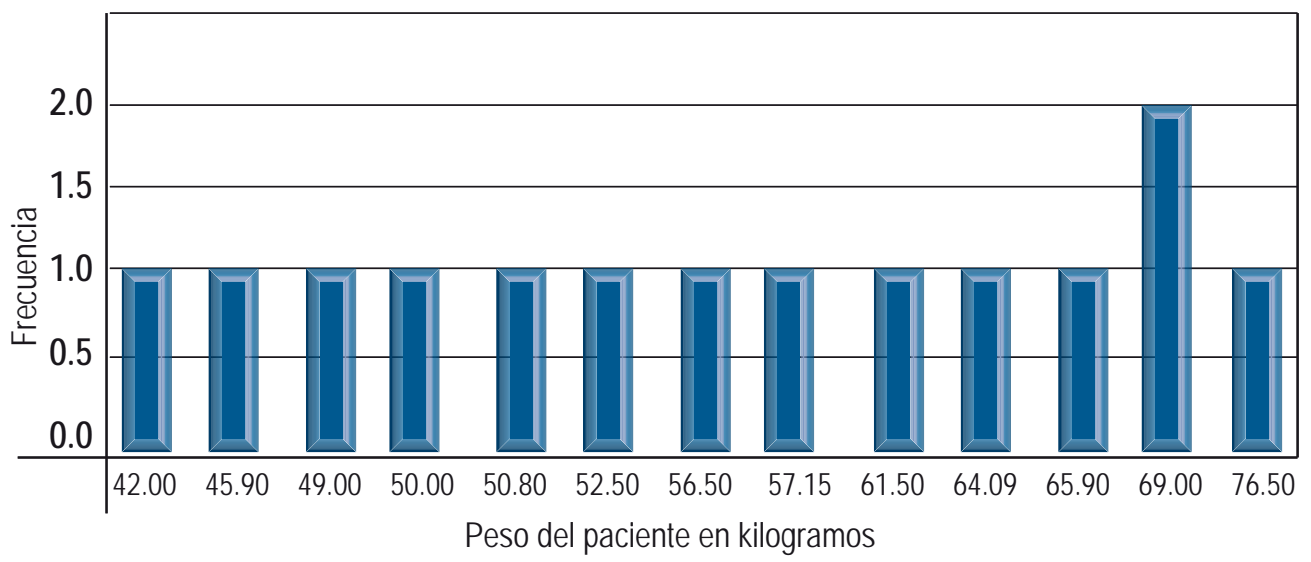

Fuente: elaboración propia.

Según el gráfico 4, el peso en kilogramos de las embarazadas indica que en su mayoría predomina el rango de peso de 42 a $76,5 \mathrm{~kg}$; convirtiéndose en una población vulnerable a desarrollar obesidad y, en consecuencia, diabetes gestacional. 
Gráfico 5. Edad gestacional en semanas

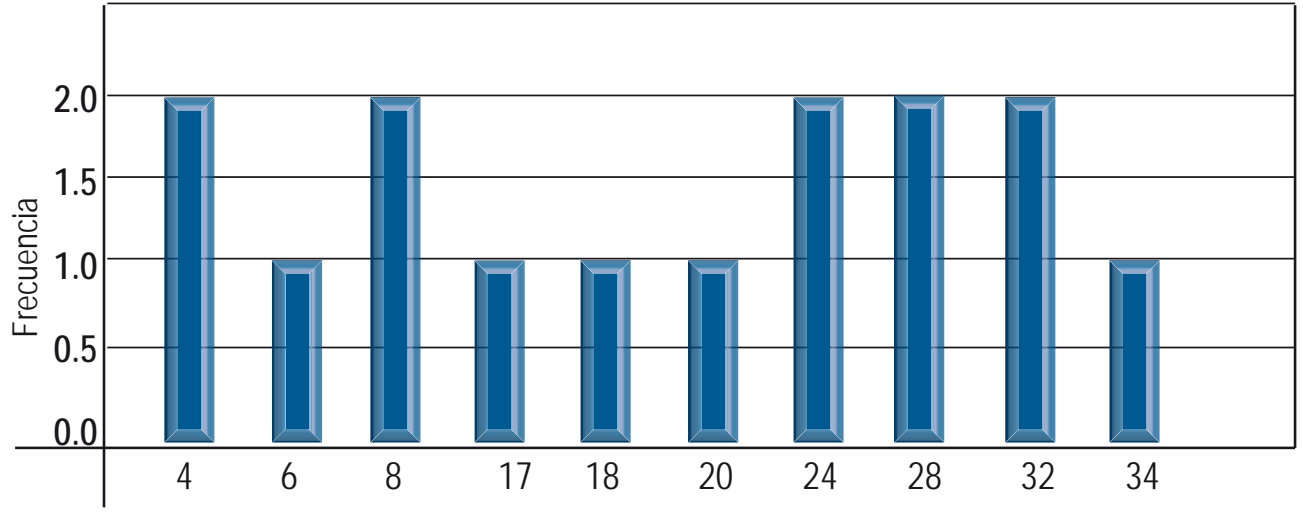

Fuente: elaboración propia.

El gráfico 5 muestra que la edad gestacional, en la mayor parte de las embarazadas (un total de 8), se ubica en el primer trimestre de embarazo y el resto, que son la minoría, están en su segundo trimestre de embarazo.

Gráfico 6. Presión arterial de las mujeres embarazadas

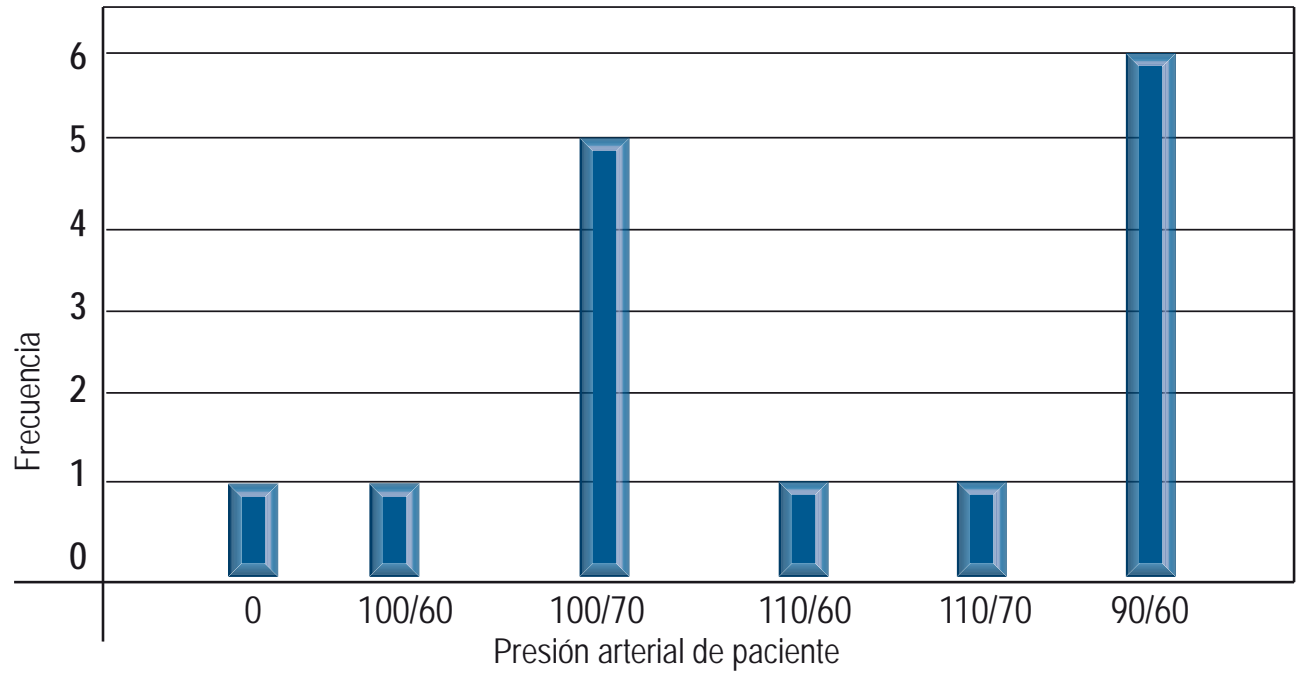

Fuente: elaboración propia.

El gráfico 6 señala que el valor de la presión arterial de las embarazadas se ubica entre 90/60 mmg y 110/70 mmg; siendo ambos parámetros los de mayor representación. Se concluye que en su mayoría no presentan riesgo de padecer hipo/hipertensión. 
Gráfico 7. Cantidad total de embarazos

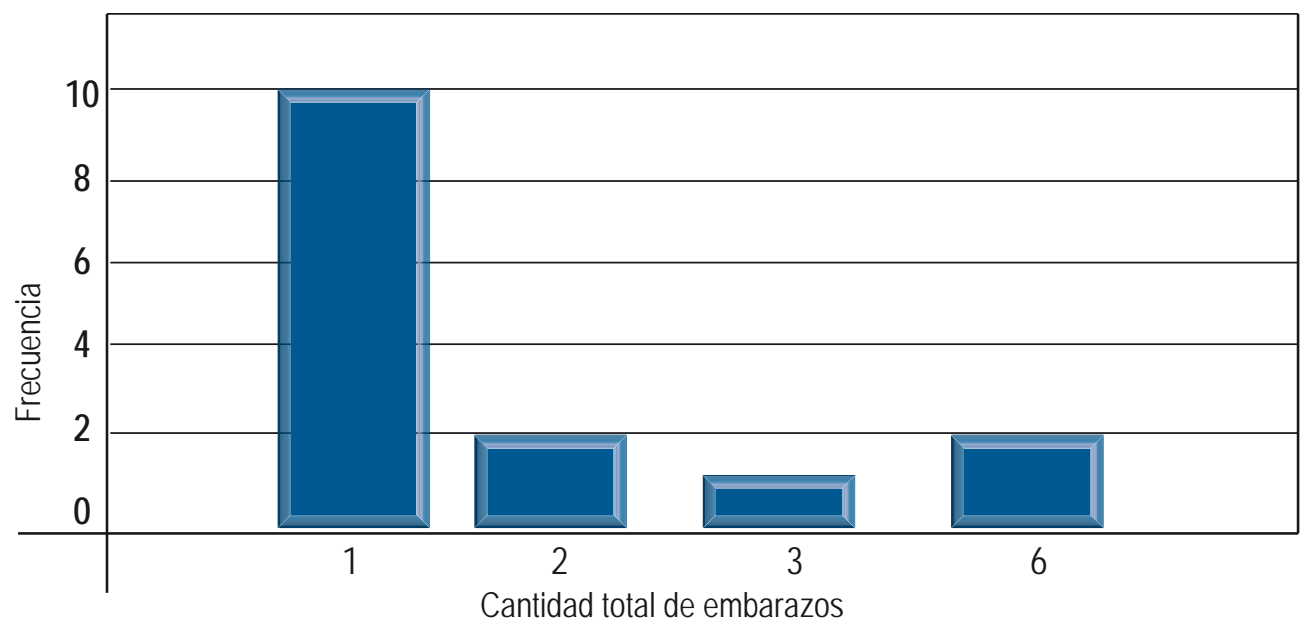

Fuente: elaboración propia.

Según el gráfico 7, el mayor porcentaje de las embarazadas son primigestas (10), el resto son multíparas (6); en consecuencia, según el dato anterior, se pueden tomar medidas preventivas con relación a la diabetes gestacional.

Gráfico 8. Alimentos consumidos por las embarazadas

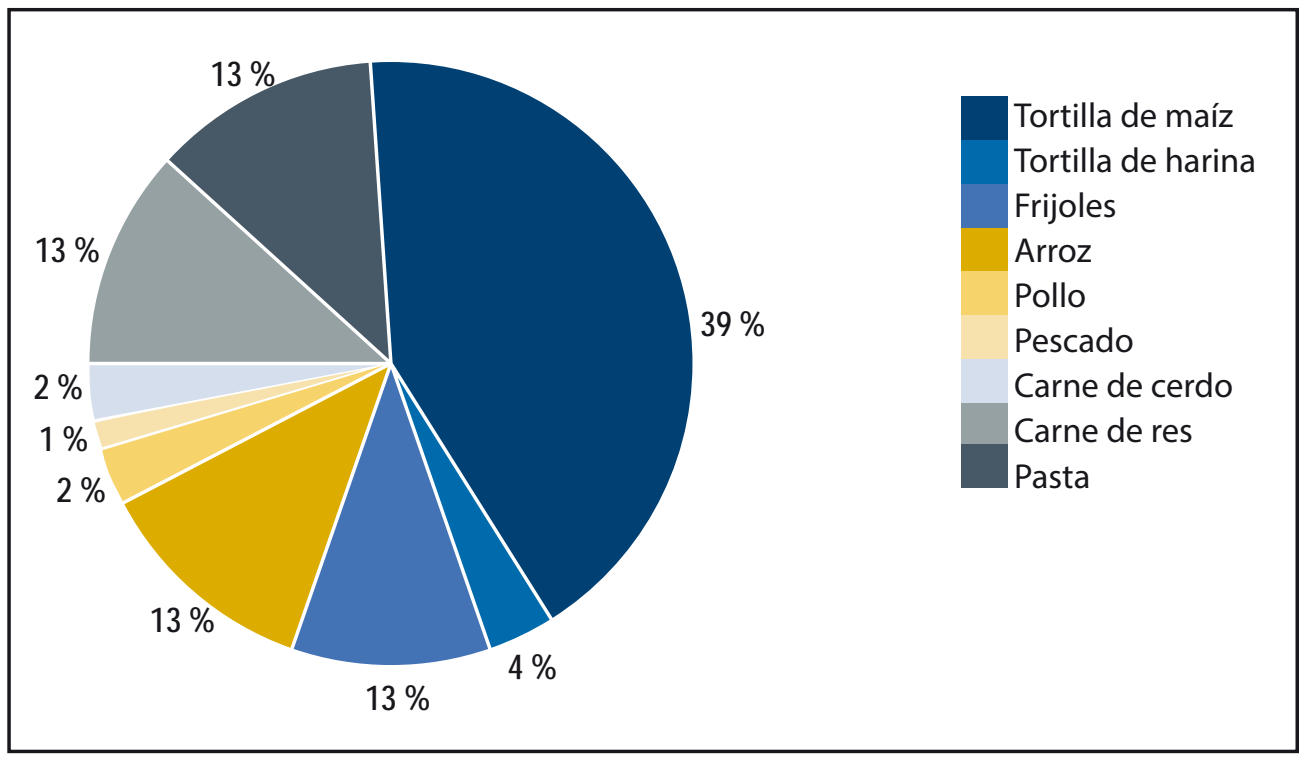

Fuente: elaboración propia. 
El gráfico 8 muestra que la alimentación en las embarazadas es predominantemente alta en carbohidratos, lo cual se convierte en un fuerte factor a futuro en el desarrollo o padecimiento de diabetes gestacional.

Gráfico 9. Bebidas o sustancias consumidas por las pacientes

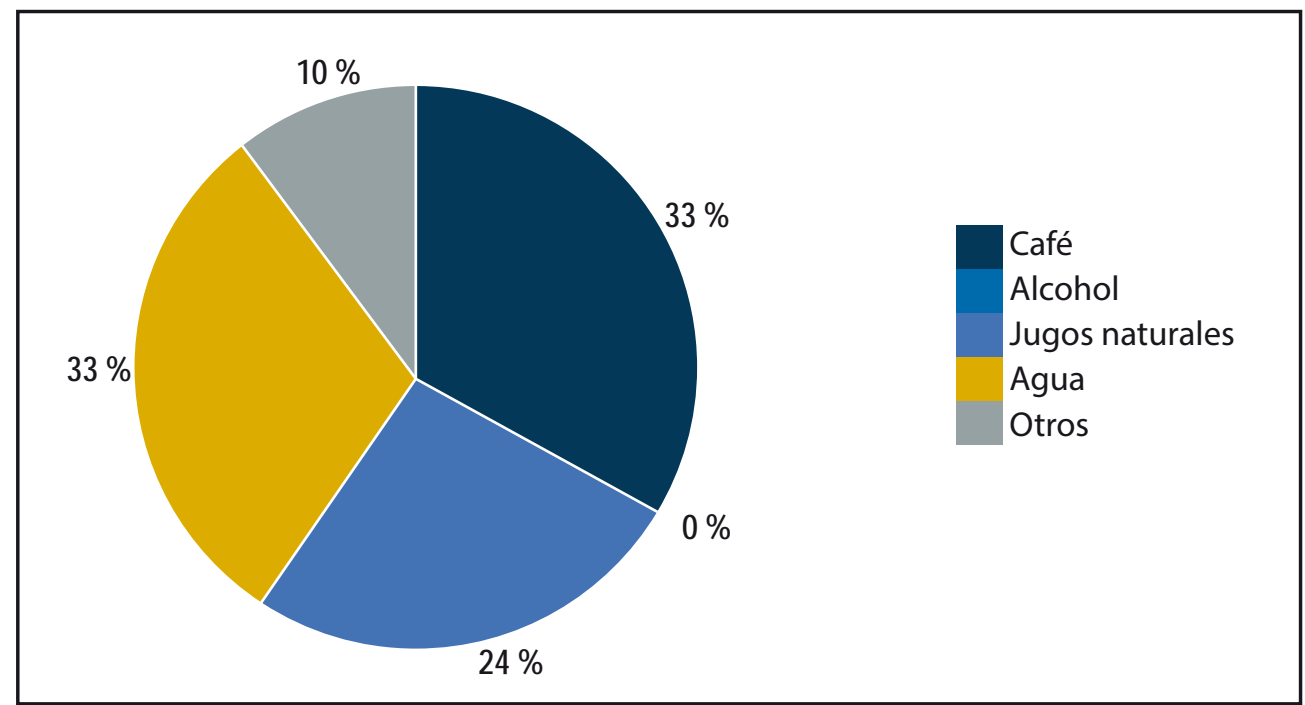

Fuente: elaboración propia.

Según el gráfico 9, las bebidas y sustancias de regular consumo en las embarazadas indica que la de mayor predominancia es el agua, seguida de la cafeína (café). Al respecto, es preciso mencionar que el uso de sustancias tóxicas, como el alcohol, no fue reportado por ellas.

\section{DISCUSIÓN}

1. El $80 \%$ de las mujeres embarazadas que reciben atención ambulatoria en el Centro de Salud Alonso Suazo no conocen sobre la diabetes gestacional.

2. Los factores de riesgo que promueven el desarrollo de diabetes gestacional y más conocidos por las embarazadas son 4: el nivel de azúcar en sangre, sobrepeso, alimentación y sedentarismo. 
3. La edad de las embarazadas que reciben atención ambulatoria oscilan en un rango de 17 a 38 años de edad. Predominando las siguientes edades: 17 a 18 y 23 a 31 años.

4. El peso en kilogramos de las embarazadas indica que un mínimo porcentaje de ellas se encuentra con sobrepeso, siendo este un factor para el desarrollo de diabetes gestacional.

5. La edad gestacional y las semanas brindadas por las embarazadas se pueden ubicar, en su mayoría, en el primer trimestre y el resto de ellas en el segundo trimestre.

6. La presión arterial de las embarazadas oscila entre un rango de 90/60 mmg -110/70 mmg, lo que significa que ninguna de ellas padece de hipo/hipertensión.

7. De las 15 embarazadas de esta investigación, 10 son primigestas y el resto de ellas multíparas, lo que puede ser muy útil para tomar medidas de prevención sobre la diabetes gestacional.

8. La alimentación predominante en las embarazadas es alta en carbohidratos, por lo que se convierten en una población vulnerable para el desarrollo de la diabetes gestacional.

9. Entre las bebidas y sustancias que consumen regularmente las embarazadas, el primer lugar lo obtuvo el agua, seguida de la cafeína (café); esto es favorable para su salud, sin dejar de mencionar que no existe el uso de sustancias tóxicas.

\section{CONCLUSIONES}

Al realizar esta investigación con las embarazadas que reciben atención ambulatoria en el área de ginecología del Centro de Salud Alonso Suazo, se observa la necesidad de educación en dicha población, con respecto al tema educativo sobre la diabetes gestacional, ya que el $80 \%$ de los datos estadísticos indica que en su mayoría no conocen sobre la enfermedad.

Esto se atribuye a la falta de información que brinda el sector salud, además de no contar con materiales informativos que pueden ser de gran ayuda, sin excluir a las 
embarazadas que en gran parte se atribuye el poco interés de su parte en saber acerca de todo lo relacionado a su estado de embarazo; muchas de ellas sabiendo las consecuencias, no toman medidas preventivas, convirtiéndose en población vulnerable próxima a desarrollar diabetes gestacional.

Por lo tanto, es necesario hacer énfasis en la prevención de casos nuevos, lo que contribuirá a reducir las tasas de morbilidad, incidencia y prevalencia de las antes mencionadas, empleando formas fáciles y prácticas para hablar sobre la diabetes gestacional, haciendo uso del personal de salud, brindando charlas educativas 0 realizando material didáctico como volantes, murales o hacer uso de los diferentes medios de comunicación (radio, televisión, medios impresos).

\section{AGRADECIMIENTOS}

A nuestra asesora Perla Simmons, por brindar sus conocimientos, experiencias vividas y asesorías durante todo este trayecto. Igualmente, a las autoridades de salud que laboran en el Centro de Salud Alonso Suazo por su apoyo incondicional y a las embarazadas que fueron participes de la investigación por su disponibilidad, voluntad y apoyo en el llenado de encuestas.

\section{BIBLIOGRAFÍA}

Almirón, M.; Gamarra, S. y Gonzales, M. (2005). Diabetes gestacional. Posgrado de la VI Cátedra de Medicina ,152. Recuperado de: http://www.med.unne.edu.ar/revista/revista152/7_152.pdf

Campo, M. y Estrada, G. (2008). Factores de riesgo para diabetes gestacional en población obstétrica. Revista CES Medicinas, 22(1). Recuperado de: http://www.ces.edu.co/

World Health Organization. (2014). Global health estimates: deaths by cause, age, sex and country, 2000-2012. Geneva. 